\title{
Attentional capture by abrupt onsets and feature singletons produces inhibitory surrounds
}

\author{
JEFFREY R. W. MOUNTS \\ State University of New York, Geneseo, New York
}

\begin{abstract}
Two experiments found that form discriminations to a target item were inhibited when the target appeared adjacent to an attentionally salient item. Experiment 1 manipulated the attentional salience of an irrelevant color singleton through the attentional set adopted by the subjects. Color singletons captured attention when the target was itself a feature singleton, but not when the target was defined as a conjunction of features. Attentional capture was accompanied by an inhibitory region (i.e., slowed target reaction times), which dissipated with distance from the color singleton. In Experiment 2, the attentional salience of abrupt onsets and color singletons was compared. Irrelevant abrupt onsets captured attention, whereas irrelevant color singletons failed to capture attention. Again, an inhibitory region surrounded the attentionally salient abrupt onsets, but not the color singletons. The results are discussed in the context of current models of visual spatial attention and suggest a distinction between attentional preparation and attentional selection.
\end{abstract}

Visual selective attention endows selected objects with a special processing status within the visual system. Empirical evidence suggests that visual selective attention enhances the speed (e.g., Eriksen \& Hoffman, 1972; Henderson \& Macquistan, 1993; Posner, Snyder, \& Davidson, 1980) and accuracy (e.g., Cheal \& Lyon, 1992; Downing, 1988; Hawkins et al., 1990; Nakayama \& Mackeben, 1989) of perceptual discriminations of the selected objects. However, the mechanisms by which attentional selection produces these benefits are still under debate.

One popular metaphor for the operation of selective attention is that of a filter: At the locus of attention, the flow of visual information is enhanced relative to unattended locations (see Cheal, 1997, for a review). Some theorists have proposed that the attentional filter normally takes a gradient structure (e.g., Cheal, Lyon, \& Gottlob, 1994; LaBerge, 1995; LaBerge \& Brown, 1989), with the rate of information flow dropping monotonically with the distance from the attentional focus (or foci). Henderson and Macquistan (1993) used a cuing paradigm to explore the spatial distribution of attention. In their experiments, a display containing a location precue (a line underscoring one of the possible stimulus positions) was presented for $100 \mathrm{msec}$ and was then replaced by a display containing a target letter. Even though the location precue was uncorrelated with the subsequent location of the target letter, Henderson and Macquistan found that as the precue-letter separation increased, letter identification accuracy decreased and response latency increased.

This research was supported by grants from the Geneseo Foundation. The author thanks Jennie Herbert and Robin Tomaselli for help in data collection and analysis, and Marvin Chun, Brad Gibson, and Steve Yantis for useful comments on an earlier draft of this manuscript. Correspondence should be addressed to J. R. W. Mounts, Department of Psychology, SUNY Geneseo, Geneseo, NY 14454 (c-mail: mounts@geneseo.edu).
They interpreted these results as indicating that attention was distributed as a gradient, peaked at the location of the precue and diminishing with distance from the peak. LaBerge and colleagues (e.g., LaBerge \& Brown, 1989; LaBerge, Carlson, Williams, \& Bunney, 1997) used a different paradigm to explore the spatial distribution of attention. LaBerge and Brown (1989) used a two-to-go paradigm, in which subjects had to monitor two successive stimulus displays (letter strings) for predefined targets. The first stimulus (with a centrally located target) was used to "set" the subjects' attentional focus, and response time (RT) following the second stimulus was used to gauge the efficiency of the visual processing of the second target. LaBerge and Brown found that RTs were fastest when the two targets both appeared at the same location, with RT rising with increased separation between the two target locations. They concluded that these data were best explained by a gradient model of attentional allocation: Processing efficiency tapers as we move away from the focus of attention. Moreover, LaBerge and Brown demonstrated that manipulating the spatial characteristics of the attention setting stimulus (i.e., width) leads to changes in the shape (breadth) of the attentional gradient. LaBerge et al. (1997) offered further evidence supporting this gradient-based explanation and ruled out moving spotlight explanations of these data.

While there is much data supporting a gradient filter conceptualization of attention, other research suggests that selective attention takes a very different form. Specifically, recent behavioral data indicate that visual processing is inhibited at locations neighboring an attended object. For example, Cave and Zimmerman (1997) asked subjects to perform a visual search task while monitoring the appearance of a probe. They found that simple RTs to the detection of a probe became slowed near the target as the detection efficiency of the target increased through 
practice. Bahcall and Kowler (1999) examined the accuracy with which observers could identify two letters at precued locations within a circular array of letters. As separation between the letters decreased, so too did the identification accuracy. Both Caputo and Guerra (1998) and Mounts (2000) used a variant of the attentional capture paradigm to explore these localized inhibition effects. Caputo and Guerra had observers make line length discriminations of a line embedded within one type of singleton (e.g., form), while a second irrelevant singleton distractor (e.g., color) was presented elsewhere in the display. They found that as the distance between the target and distractor decreased, the line length discrimination threshold increased. Similarly, Mounts asked observers to discriminate the identity of a target embedded among an array of elements. The distractors were all block $8 \mathrm{~s}$, and the target was a form singleton created by removing the top or the bottom of one of the block $8 \mathrm{~s}$ (yielding a block A or an upside-down A). Subjects' target discrimination performance was measured as a function of the distance between a color or orientation singleton also embedded within the display. Stimuli were presented briefly $(<100 \mathrm{msec})$ and masked. As the distance between the attentionally salient distractor (color or orientation singleton) and the target item decreased, so too did target discrimination performance. These studies suggest that attentional facilitation of one object comes at a cost to the processing of neighboring objects. One explanation is that attention actively inhibits the processing of items surrounding the focus of attention (e.g., Luck, Girelli, McDermott, \& Ford, 1997). According to Luck et al.'s ambiguity resolution theory, this inhibition is necessary because multiple objects might fall within the relatively large receptive fields of neurons in extrastriate areas. This could potentially lead to ambiguity in the coding of features from any single object (see also Desimone \& Duncan, 1995). Inhibiting the processing of objects near the attended object would alleviate this ambiguity problem. Bahcall and Kowler (1999) offered a space-based, resource allocation explanation, suggesting that, at the attended location, processing strength is increased by borrowing resources from neighboring regions. Regardless of the precise mechanisms driving this phenomenon, these data suggest that attentional selection of an object is accompanied by inhibited perceptual processing of objects spatially near the selected object.

The attentional capture paradigm appears to be an ideal methodology within which to explore the localized inhibition effects described above. In a typical attentional capture task, subjects search for and make a speeded perceptual discrimination regarding a target embedded in an array of distractors (detection and discrimination tasks are both used regularly). Also present in the display is an object possessing a feature that is a potential attractor of attention. Attentional capture is defined as the unintentional deployment of attention to the object possessing this feature. The allocation of attention to an object has been operationalized in several ways, depending on the task (discrimination or detection). Most definitions focus on trials in which the target itself possesses the unique feature. For discrimination tasks, attentional capture is often operationalized as facilitated RTs when the target itself possesses the feature under consideration, as opposed to one of the distractors (e.g., Folk \& Remington, 1998; Folk, Remington, \& Johnston, 1992). However, Theeuwes (1991, 1992) defined attentional capture as the slowing of RTs when an attentionally salient distractor (e.g., feature singleton) is included in the display (but see Folk \& Remington, 1998). For detection tasks, attentional capture is said to occur when target detection RT is insensitive to display size when the target possesses the attentionally salient feature but increases with display size when this feature resides elsewhere in the display (e.g., Yantis \& Egeth, 1999; Yantis \& Jonides, 1984).

If attentional selection leads to the inhibition of neighboring objects (e.g., Caputo \& Guerra, 1998; Mounts, 2000 ), this suggests a third way to identify attentional capture: We would expect greater latency in target discriminations when the target is near the object capturing attention as opposed to when these two objects are more distant. Such results would also generalize the localized inhibition observed by Caputo and Guerra and by Mounts to discrimination RTs. ${ }^{1}$ This study explores how the capture of attention by an attentionally salient feature impacts the latency of perceptual discriminations to other objects in the display. In addition, the impact of task demands and subjects' attentional set on an item's attentional salience is examined.

\section{EXPERIMENT 1 Color Singletons and Attentional Set}

Caputo and Guerra (1998) and Mounts (2000) found decrements in perceptual performance near feature singletons. The data suggested that the feature singletons captured attention and that attending to the feature singleton inhibited the perceptual processing of neighboring elements. The conditions under which feature singletons capture attention have been the focus of recent research (e.g., Bacon \& Egeth, 1994; Folk \& Remington, 1998; Folk et al., 1992; Theeuwes, 1992). Bacon and Egeth concluded that feature singletons capture attention when observers adopt what they call a singleton detection mode - attention is guided to unique features in the visual scene. However, if observers adopt what they term a feature search mode, feature singletons do not capture attention. They demonstrated that the attentional control settings used by subjects may be dictated by task demands. If the target can be identified on the basis of a unique feature (i.e., it too is a feature singleton), subjects will likely adopt the singleton detection strategy, and a salient distractor feature singleton will capture attention. If the target cannot be identified on the basis of a single unique feature, subjects will likely adopt a feature search mode, and a singleton distractor will not capture attention. In the experiments of Caputo and Guerra (1998) and Mounts 
(2000), the target was defined as a feature singleton; therefore, the observers likely adopted a singleton search mode, leading to attentional capture by the distractor singleton. In Experiment 1 of the present study, stimulus displays included an irrelevant color singleton distractor. Task demands were manipulated to alter the attentional salience of this color singleton. If subjects adopt a singleton search mode when searching for the target, a color singleton distractor should capture attention, and an inhibitory region is predicted to surround the color singleton under these conditions. If subjects adopt a feature search mode when searching for the target, the color singleton should not capture attention, and no inhibitory region should be observed.

\section{Method}

Subjects. Sixteen students ( 13 women and 3 men) from the State University of New York at Geneseo participated in the experiment for course credit. Each session lasted approximately $30 \mathrm{~min}$. All subjects reported normal or corrected-to-normal acuity and color vision.

Apparatus and Stimuli. Stimuli were presented on an AppleVision 1710 monitor set to a resolution of $1,152 \times 852$ pixels with a frame rate of $75 \mathrm{~Hz}$. The subjects were seated $57 \mathrm{~cm}$ from the display, with head stabilization aided through the use of a chin-andforehead rest. Responses were made using a PsyScope Button Box (New Micros, Dallas). All sessions were conducted in a darkened, sound-attenuating booth.

Stimuli were block figure $8 \mathrm{~s}$, each stimulus subtending $1.6^{\circ} \times$ $1.0^{\circ}$; the individual line segments used to create the stimuli were $0.24^{\circ}$ in width. The target stimuli were the block letters $\mathrm{E}$ and $\mathrm{H}$, created by removing the appropriate segments from block $8 \mathrm{~s}$. The stimuli were either red $\left(x=.47, y=.46 ;\right.$ luminance, $\left.11.89 \mathrm{~cd} / \mathrm{m}^{2}\right)$ or green $\left(x=.35, y=.54\right.$; luminance, $\left.11.90 \mathrm{~cd} / \mathrm{m}^{2}\right)$ presented against a gray $\left(x=.28, y=.29\right.$; luminance, $\left.1.96 \mathrm{~cd} / \mathrm{m}^{2}\right)$ background. Stimuli appeared at eight stimulus locations, spaced equally along an imaginary circle with a radius of $5.0^{\circ}$. One of the display elements was one of the two target letters ( $\mathrm{E}$ or $\mathrm{H})$, and the other seven elements were distractors. The subject's task was to respond as quickly as possible to the identity of the target letter by pressing the right $(\mathrm{E})$ or left $(\mathrm{H})$ key on the response box.

Conditions and Procedure. The subjects completed four blocks of 144 trials, with each block preceded by 20 practice trials. Two blocks of trials were run in the singleton target condition, and the other two blocks of trials were run in the conjunction target condition. For each subject, the ordering of the four blocks of trials was set using a Latin square. The subjects were instructed to respond as quickly as possible while maintaining an accuracy rate above $90 \%$. Incorrect responses were signaled by a computer beep. Speed and accuracy feedback was provided every 48 trials.

Singleton target condition. In this condition, the seven distractors were all block $8 \mathrm{~s}$, making the target $(\mathrm{E}$ or $\mathrm{H})$ a form singleton (see Figure 1A). This should allow the subjects to adopt a singleton detection strategy, rendering the irrelevant color singleton attentionally salient. Within the set of 144 trials, the target appeared equally often at each of the eight possible locations. The color singleton also appeared equally often at each of the eight possible locations and was withheld on $1 / 9$ of the trials to create a control condition. The location of the target and the location of the color singleton distractor were uncorrelated, with each possible target/distractor location pairing occurring twice (once for each target value). Consequently, the target was itself a color singleton on $1 / 9$ of the trials. In one block of trials, a green color singleton was embedded among the red stimuli; in a second block of trials, a red color singleton was embedded among green stimuli.

Conjunction target condition. In this condition, the target ( $\mathrm{E}$ or $\mathrm{H}$ ) was presented within a heterogeneous array of seven other let-

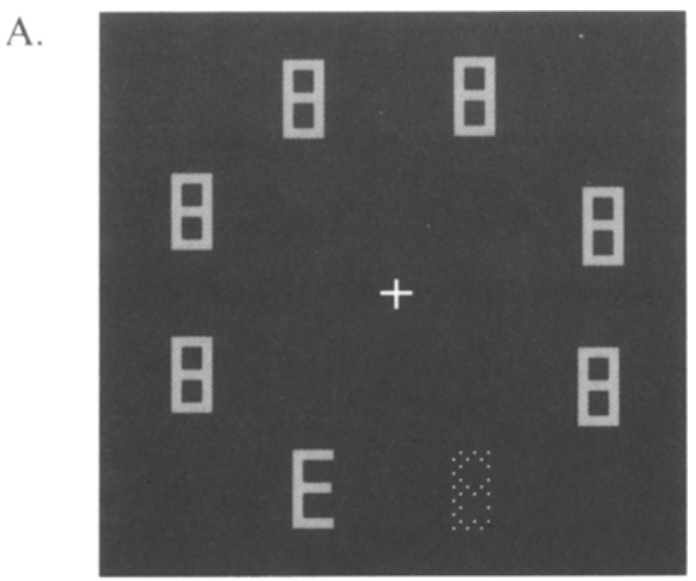

Target Display: Until Response

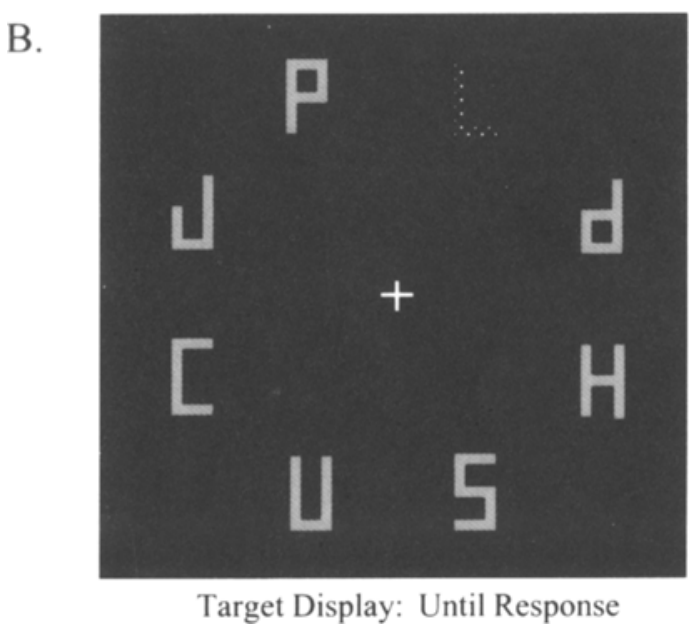

Figure 1. Sample stimulus displays for the singleton target condition (Panel A) and the conjunction target condition (Panel B) in Experiment 1 . The subjects searched for the targets $E$ or $H$. In the singleton target condition, the display contained seven block 8 and the target. In the conjunction target condition, the display contained seven distractor lefters $(d, L, P, J, C, U$, and $S$ ) and the target letter. In both conditions, one of the items differed in color from the other elements (i.e., the color singleton). The display remained until a response was made.

ters (see Figure 1B). As a result, there were no unique features that distinguished the target set ( $E$ and $H)$ from the distractor set $(d, U$, P, S, L, J, and C). Consequently, the subjects should be forced into a feature-detection strategy, and color singletons should not capture attention. The breakdown of target and distractor locations across trials was identical to that of the singleton target condition. Again, in one block of trials a red color singleton was embedded among green items; in a second block of trials, a green color singleton appeared among red items.

\section{Results and Discussion}

In both experiments reported in this article, outliers were trimmed from the RT data by excluding trials in which responses were faster than $300 \mathrm{msec}$ or more than 
3 standard deviations above the mean RT for a given subject in a given condition This led to the rejection of less than $2 \%$ of the trials within each of the experiments. In addition, RTs from incorrect trials were excluded from the analysis. Error rates were low across all experiments $(<3 \%)$ and paralleled the RT data; error rates were not analyzed further.

The RT data (relative to the control condition) for the singleton target and conjunction target conditions are shown in Figure 2. These data were analyzed using a $2 \times$ 5 analysis of variance (ANOVA), with the repeated measures factors of condition (singleton target vs. conjunction target) and target-distractor separation. The ANOVA revealed no main effects of condition type $[F(1,19)=$ 2.47 , n.s.] or target-distractor separation $[F(4,76)<1]$. However, the condition type $\times$ target-distractor separation interaction was statistically reliable $[F(4,76)=9.42$, $p<.001]$.

In order to determine whether the color singletons captured attention, planned comparisons contrasted trials in which the target and the color singleton were the same object (i.e., the target was a color singleton) with trials in which the target and the color singleton appeared at different locations. As can be seen in Figure 2, target performance at the singleton location was faster than at other locations in the singleton target condition $[F(1,19)=$ $29.01, p<.001]$, whereas the decrement in performance at the singleton location relative to other target locations in the conjunction target condition approached statistical significance $[F(1,19)=3.28, p=.08]$. This pattern suggests that the color singleton captured attention in the singleton target condition, whereas the singleton was fil-

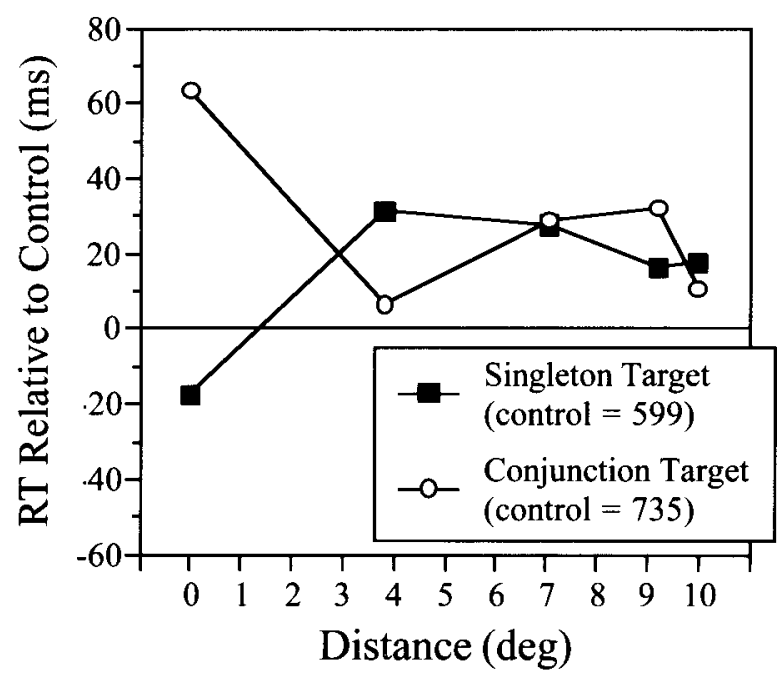

Figure 2. Target discrimination reaction times (RTs) for the singleton target and conjunction target conditions in Experiment 1. The RTs are plotted as the difference from control (no color singleton present) in each of the conditions. The control RTs are shown in the legend for each of the two conditions. A distractor-target separation of 0 indicates that the target was the color singleton. tered out in the conjunction target condition. Planned contrasts also compared performance at the color singleton location with performance in the control condition. In the singleton target condition, target performance at the color singleton location was facilitated relative to control $[F(1,19)=4.93, p<.05]$, whereas it was slowed relative to control in the conjunction search condition $[F(1,19)=$ $7.90, p<.05]$.

An examination of Figure 2 suggests that, for the singleton target condition, RTs were slowest when the target was adjacent to the color singleton and became faster as the target-distractor separation increased. These conclusions were confirmed using a linear contrast for the nonzero distances (weights were adjusted to reflect the unequal steps in distance). In other words, target RTs in the singleton target condition were slowed when the target was adjacent to the distractor and became faster with increasing separation between the singleton and the distractor [linear contrast, $F(1,19)=8.39, p<.01$ ]. In the conjunction target condition, this same linear contrast failed to reveal an effect $[F(1,19)<1]$. These results suggest that when color singletons capture attention, an inhibitory region surrounds the attended singleton.

The task demands in the singleton target condition were such that an irrelevant color singleton should have captured attention. This prediction was confirmed: RTs to targets appearing at the color singleton location were speeded relative to the control condition as well as to trials in which the target appeared at a noncolor singleton location. Furthermore, this attentional capture by the color singleton was accompanied by an inhibitory region surrounding the irrelevant color singleton distractor. This suggests that attentional selection of an item is accompanied by the inhibition of the processing of neighboring stimuli. These form discrimination RT data mirror line length threshold data of Caputo and Guerra (1998) and the form discrimination sensitivity data of Mounts (2000). In all cases, perceptual performance was hampered near attentionally salient distractors.

In contrast, the conjunction target condition revealed no evidence of capture by the color singleton, nor an inhibitory region. This finding underscores the attentional basis of the inhibitory region observed in the singleton target condition: The presence of an inhibitory region surrounding a color singleton is dependent on the attentional salience of the color singleton. As proposed by Bacon and Egeth (1994), this attentional salience is determined in part by the attentional set of the subject. When the subjects engaged in a conjunction search, irrelevant color singletons had a low attentional salience and failed to capture attention. Indeed, the data suggest that, as opposed to capturing attention, the color singleton may have been filtered out during the search for the target. Egeth, Virzi, and Garbart (1984) and Kaptein, Theeuwes, and van der Heijden (1995) found that, in a conjunction search, subjects could restrict their search to a set of objects possessing a specific color. In the present experiment, the target was the color singleton on only 16 of the 
144 trials (i.e., $11 \%$ of the trials). For example, in one block of trials, the target was green $89 \%$ of the time. It appears that the subjects may have adopted a search strategy by which green items (or red in the other condition) were given greater attentional priority.

\section{EXPERIMENT 2 Abrupt Onsets Versus Color Singletons}

In Experiment 1, color singleton distractors captured attention (and were surrounded by inhibitory regions) when the target was itself a feature singleton. However, these same color singleton distractors failed to capture attention when the target was defined as a conjunction of features. This finding was interpreted as resulting from the attentional set (singleton detection vs. conjunction search) adopted by the subjects (e.g., Bacon \& Egeth, 1994). Furthermore, it was proposed that an inhibitory region surrounded the color singleton only when the task demands rendered it attentionally salient.

However, the lack of an inhibitory surround could have been due to other differences existing between the two conditions. Specifically, as shown by the RTs to the control conditions (see Figure 2), average responses in the conjunction target condition were more than $100 \mathrm{msec}$ slower than responses in the feature target condition. It is possible that an inhibitory region surrounded the color singleton in both conditions but that the additional time needed in the conjunction target condition allowed the inhibitory region to dissipate to the point that it did not affect performance. In this view, it was not the attentional salience of the color singleton that controlled the presence or absence of an inhibitory region; rather, it was the time delay that determined whether an inhibitory region was observed.

Yet another nonsalience-based explanation concerns the similarity of the distractor elements. In the singleton target condition, the distractors were homogeneous with respect to the form dimension; in the conjunction target condition, these distractors were heterogeneous with respect to form. It is not clear why such a factor might influence an inhibitory region. Duncan and Humphreys (1989) proposed that homogeneous items are easier to inhibit, and perhaps this factor might be sensitive to the distance from the attentional focus. Nonetheless, this factor was confounded with the attentional set manipulation in Experiment 1 and, as such, might account for the observed difference. However, both of these alternative explanations could be rejected if an inhibitory region were observed under conjunction search conditions (i.e., heterogeneous distractors).

While a color singleton's ability to capture attention appears to rely on the task demands, the ability of an abrupt onset to capture attention appears to be largely immune to such task demands (see Yantis, 1996, for a review). Indeed, the attentional salience of abrupt onsets appears to derive from stimulus factors, as opposed to the attentional set of the observer. For example, in Yantis and Jonides's (1984; Jonides \& Yantis, 1988) initial studies examining abrupt onsets, the tasks mandated a conjunction search strategy on the part of the subjects, similar to the conjunction target conditions in the present Experiment 1. Nonetheless, abrupt onsets reliably captured attention in these tasks, whereas color and luminance singletons did not. The task employed in Experiment 2 is very similar in nature to the original work of Yantis and Jonides. Therefore, it was predicted that abrupt onsets would capture attention even when subjects were performing a feature search for the target. Thus, it was predicted that the abrupt onsets would capture attention, and this capture would be accompanied by an inhibitory region surrounding the abrupt onset. In contrast, color singleton distractors should again fail to capture attention.

\section{Method}

Subjects. Sixteen students ( 12 women and 4 men) from the State University of New York at Geneseo participated in the experiment for course credit. Each session lasted approximately $30 \mathrm{~min}$. All subjects reported normal or corrected-to-normal acuity and color vision.

Conditions and Procedure. The color, size, and potential locations of the stimuli were identical to those used in Experiment 1. During a trial, stimulus elements were presented at five of the eight possible locations, with the restriction that at least one element appear within each quadrant of the display. The subjects completed four blocks of 144 trials, with each block preceded by 20 practice trials. Two blocks of trials were run in the abrupt onset condition; the other two blocks of trials were run in the color singleton condition. For each subject, the ordering of the four blocks of trials was set using a Latin square. The subjects were instructed to respond as quickly as possible while maintaining an accuracy rate above $90 \%$. Incorrect responses were signaled by a computer beep. Speed and accuracy feedback was provided every 48 trials.

Abrupt onset conditions. Two of the blocks of trials corresponded to the abrupt onset condition. In these blocks of trials, four of the block 8 stimuli were presented during a preview period lasting 1,000 msec. At the end of this preview period, a new element was introduced into the display (an abrupt onset). At the same time the abrupt onset appeared, the identity of the target ( $E$ or $\mathrm{H}$ ) and distractor elements (d, S, P, U) were revealed by removing the appropriate line segments from the block 8 s (see Figure $3 \mathrm{~A}$ ). This display remained until a keypress was made. Within the set of 144 trials, the target appeared equally often at each of the eight possible locations. The abrupt onset also appeared equally often at each of the eight possible locations and was withheld on $1 / 9$ of the trials to create a control condition (five instead of four stimuli were presented during the preview period). The location of the target and the location of the abrupt onset distractor were uncorrelated, with each possible target/distractor location pairing occurring twice (once for each target value). Consequently, the target was itself an abrupt onset on $1 / 9$ of the trials. In one block of trials, all stimuli were red; in a second block of trials, all stimuli were green.

Color singleton conditions. The other two blocks of trials corresponded to the color singleton condition. A display containing five elements was presented and remained until a response was made. Of the five elements, four were distractors (d, P, S, U) and one was the target (E or H). Figure 3B illustrates an example display. Across the set of 144 trials, the target appeared equally often at each of the eight possible locations. Present in $8 / 9$ of the trials was a color singleton distractor. The color singleton appeared equally often at each 
A.

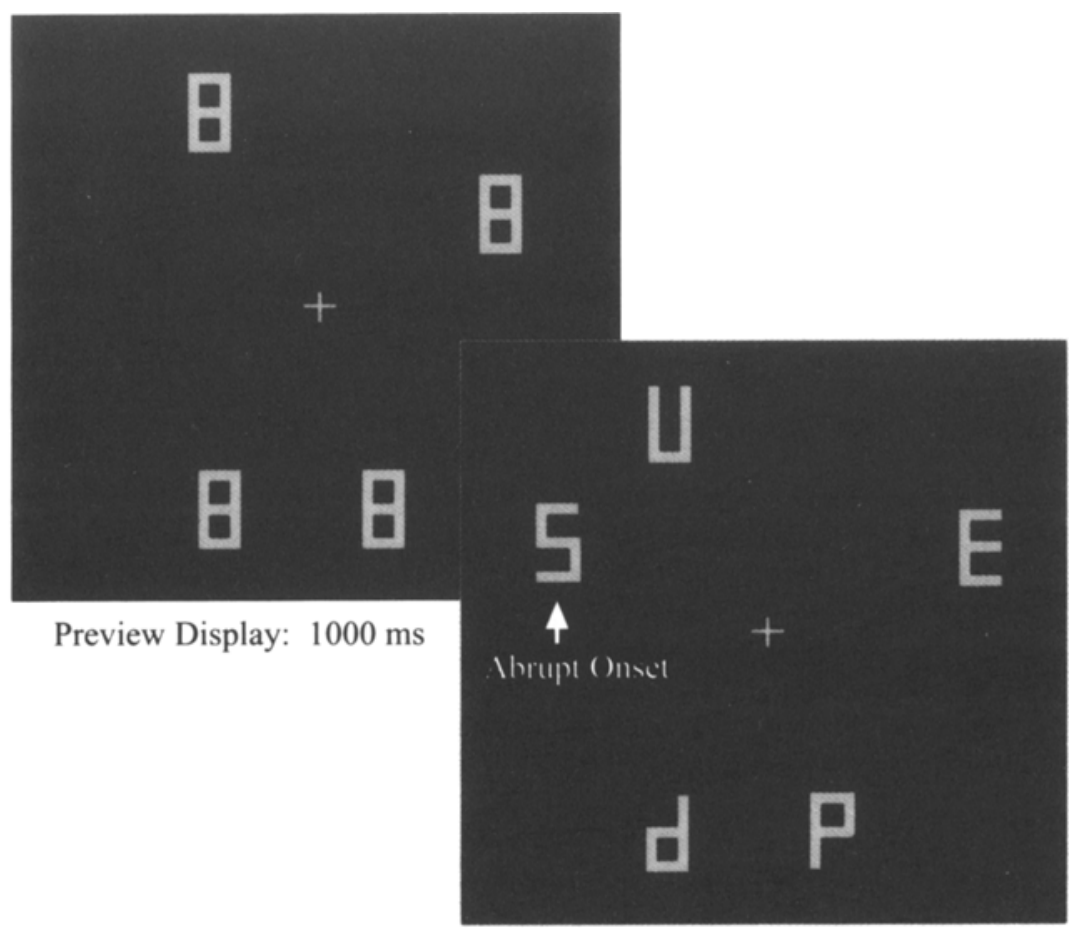

Target Display: Until Response

B.

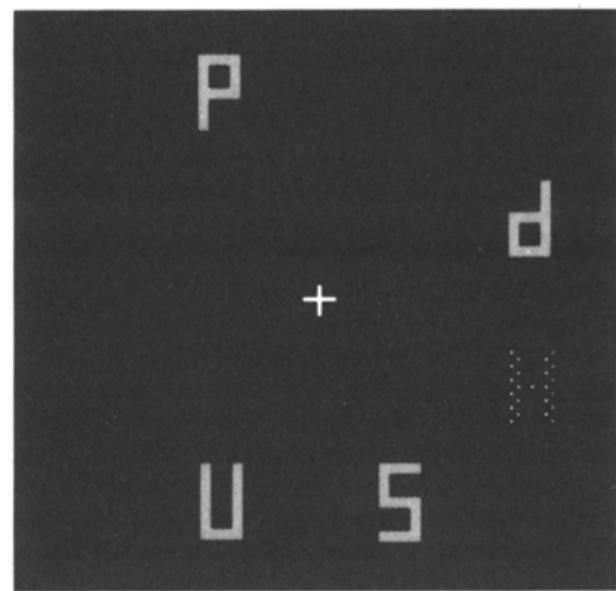

Target Display: Until Response

Figure 3. Sample stimulus displays for the abrupt onset condition (Panel A) and the color singleton condition (Panel B) in Experiment 2. The subjects searched for the targets $E$ or $H$. In the abrupt onset condition, the fixation display was followed for 1,000 msec by a display containing four place holders. The place holder display was replaced by the target display. Within the target display, an abrupt onset was introduced, and segments were removed from all of the place holder elements to create letters (including the target). This target display remained until a response was made. In the color singleton condition, a single display was used on each trial, containing both the target and a color singleton. This display remained until a response was made.

of the eight possible locations. In $1 / 9$ of the trials, a control condition was presented, with all five elements possessing the same color. The location of the target and the location of the color singleton distractor were uncorrelated, with each possible target/distractor location pairing occurring twice (once for each target value).
As a result, on $1 / 9$ of the trials, the target and the color singleton appeared at the same location (i.e., the target was a color singleton). In one block of trials, the stimuli were red and the color singleton was green; in a second block of trials, the stimuli were green and the color singleton was red. 


\section{Results and Discussion}

The RT data (difference from the control condition) were analyzed using a $2 \times 5$ ANOVA, with the repeated measures factors of distractor type (abrupt onset vs. color singleton) and target-distractor separation. The ANOVA revealed no main effects of distractor type $[F(1,15)=3.18$, n.s. $]$ or target-distractor separation $[F(4,60)=1.60$, n.s. $]$. However, the distractor type $\times$ target-distractor separation interaction was statistically reliable $[F(4,60)=12.22, p<.001]$. The RT data (relative to control) for correct responses as a function of type of distractor (abrupt onset vs. color singleton) and separation between the distractor and the target can be found in Figure 4.

As in Experiment 1, a planned comparison was conducted on trials in which the target was an abrupt onset or a color singleton versus trials in which one of the distractors was the abrupt onset or color singleton. For the abrupt onsets, we found evidence for attentional capture: RTs were faster when the target was the abrupt onset than when the abrupt onset appeared at a nontarget location $[F(1,15)=23.48, p \leq .001]$. Examining the color singleton condition, we observed a reliable slowing of RT when the target was the color singleton $[F(1,15)=14.96$, $p \leq .005]$, suggesting that no attentional capture occurred. Finally, facilitation and inhibition for targets at the distractor location was assessed by using a planned contrast to compare performance when targets appeared at the distractor location with target performance in the control condition. These contrasts revealed that responses to targets that were also abrupt onsets were speeded relative to responses in the control condition $[F(1,15)=16.57$,

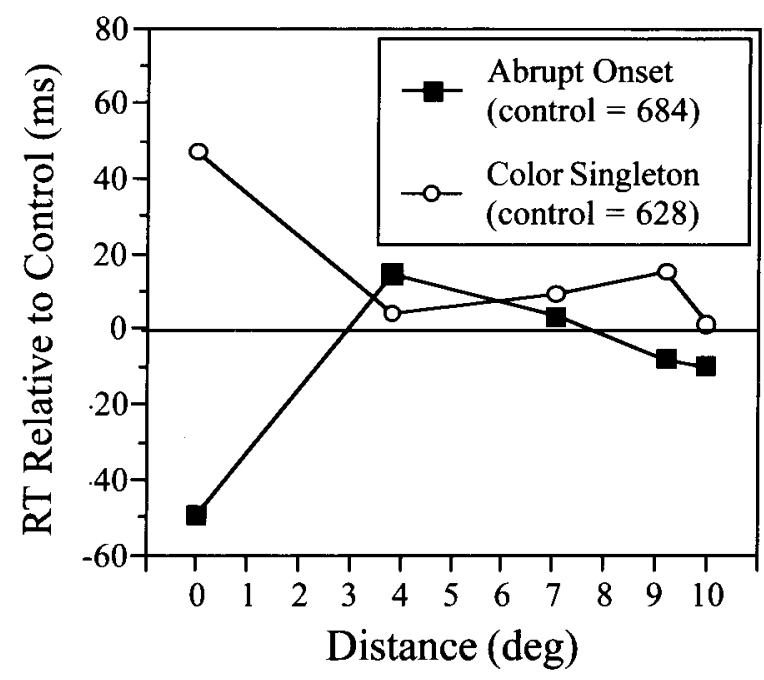

Figure 4. Target discrimination reaction times (RTs) for the abrupt onset and color singleton conditions in Experiment 2. The RTs are plotted as the difference from control (no distractor present) in each of the conditions. The control RTs are shown in the legend for each of the two conditions. A distractor-target separation of 0 indicates that the distractor and target were the same element (e.g., the target was the abrupt onset). $p \leq .001]$, whereas responses to targets that were also color singletons were reliably slower than responses in the control condition $[F(1,15)=20.80, p \leq .001]$.

Examining the RTs for the abrupt onset condition, we see that RTs were slowest when the target was adjacent to the distractor and became faster as the target-distractor separation increased, as in Experiment 1. This pattern was confirmed using the same linear contrasts employed in Experiment 1; for abrupt onsets, the linear contrast was statistically reliable $[F(1,15)=7.65, p \leq .05]$, indicating that RTs to the target speeded as separation from the distractor increased. However, this pattern was not observed in the color singleton condition $[F(1,15)<1]$.

In the abrupt onset condition, the pattern of performance was similar to that observed in Experiment 1. Target discriminations were fast at the location of the abrupt onset, slow at locations adjacent to the abrupt onset, and increased in speed as the separation between the abrupt onset and target increased. Again, this pattern suggests that the abrupt onset captured attention and that this in turn led to an inhibition of neighboring objects. Moreover, abrupt onsets captured attention under conditions promoting a conjunction search, ruling out the potential confounds in Experiment 1 related to task type and stimulus displays. These results are consistent with the notion that abrupt onsets have a stronger attentional salience, perhaps obligatorily drawing attention to their location (cf. Jonides \& Yantis, 1988; Yantis \& Hillstrom, 1994).

\section{GENERAL DISCUSSION}

Two experiments provided evidence for localized inhibitory regions related to the operation of selective attention. These inhibitory regions manifested as slowed RTs to targets presented near attentionally salient distractors. These RT data replicate and extend previous work that used perceptual sensitivity (Mounts, 2000) and threshold values (Caputo \& Guerra, 1998) as dependent variables. The findings are also consistent with findings from other paradigms suggesting that a ring of inhibition surrounds an attended object (e.g., Bahcall \& Kowler, 1999; Cave \& Zimmerman, 1997).

The present data - specifically those pertaining to color singletons-provide strong evidence that these inhibitory effects are attentionally based. In Experiment 1, the stimulus displays in the singleton target and conjunction target conditions were identical with respect to the color dimension. Consequently, sensory-based explanations of the inhibitory surround can be ruled out (at least for the color singletons), since the singleton distractors were functionally equivalent in the two conditions. The color singletons' effect on performance depended on the task demands (which presumably influenced the attentional set of the subjects). When the task demands rendered the color singleton attentionally salient (i.e., the singleton target condition), inhibition surrounded the color singleton. When task demands were changed such that the color singleton should have low attentional salience (i.e., 
the conjunction target condition), no inhibition surrounded the color singleton, and the color singleton itself appeared to be inhibited. Thus, the inhibitory surround appears to be a consequence of attentional selection and, as such, can be used as an indicator of attentional capture. Although such an attentional dissociation was not attempted for the abrupt onset distractors, the similar pattern of inhibition suggests the operation of like mechanisms in the two cases.

\section{Attention: Gradient of Facilitation Versus Inhibitory Surround}

Strong evidence is accumulating that attentional selection of one object results in the inhibition of perceptual processing for neighboring objects. In addition to the empirical support from behavioral studies noted above, such an attentional selection mechanism has support from physiological studies (e.g., Chelazzi, Miller, Duncan, \& Desimone, 1993; Moran \& Desimone, 1985, Motter, 1993) as well as the behavioral studies previously reviewed. Furthermore, an examination of the structure and physiology of the visual system suggests the need for such a selection mechanism (e.g., Desimone \& Duncan, 1995; Desimone, Wessinger, Thomas, \& Schneider, 1990; Luck et al., 1997). However, the data in support of neighborhood inhibition appear to conflict with other data within the behavioral literature, data pointing to graded facilitation surrounding an attended location (e.g., Downing \& Pinker, 1985; Henderson \& Macquistan, 1993; LaBerge \& Brown, 1989; LaBerge et al., 1997). While there appears to be a discrepancy between studies supporting a gradient of facilitation and those suggesting an inhibitory surround, a closer analysis suggests that the experimental paradigms producing these data may be tapping into distinct attentional mechanisms.

LaBerge et al. (1997; see also LaBerge, 1995) make the distinction between preparatory attention and selective attention. They proposed that both manifested as a gradient of increased activity centered on the attended location. Preparatory attention (based on an expectation of target location) creates a relatively broad distribution of facilitation, whereas selective attention (selecting the target once it is presented) is characterized by a relatively narrow distribution centered on the selected object- the width of the distribution determined by the size of the selected object. The observed gradients in perceptual facilitation associated with spatial cuing studies and in LaBerge's studies can be readily explained by the preparatory attention gradient.

While the preparatory attentional gradient can account for the gradations in facilitation across space in the spatial cuing paradigm, it is inconsistent with the inhibitory regions that surround attended objects in this study and other studies. It is possible that this discrepancy arises because the studies that find an inhibitory surround are not tapping into this preparatory attention mechanism but rather reveal the operation of the selective attention mechanism. In the studies demonstrating an inhibitory surround, an attentionally selected object is always present simultaneously with the target (e.g., the color singleton was present together with the target in the present study). As a result, performance to the target gauges the consequence of attentional selection of the distractor. In studies that observe a gradient of facilitation, the attentiondirecting stimulus is typically removed prior to the presentation of the target. For example, in spatial cuing studies, it is standard to remove the cue prior to the presentation of the target to avoid the cue masking the target (see Chastain, 1992; Cheal, 1993). In the paradigm popularized by LaBerge and colleagues, the attention-setting stimulus is also removed prior to the presentation of the target display. In both cases, the removal of the object directing the attentional focus may disengage the attentional selection mechanisms (those selecting the attended object and producing the inhibitory surround). Consequently, these studies may tap only the preparatory attentional mechanisms (those increasing the processing strength to a given region of space). According to this account, a distinction needs to be made between preparatory and selective mechanisms of attention, as proposed by LaBerge et al. (1997). However, the present data suggest that selective attention mechanisms lead to an inhibition of neighboring objects, as opposed to merely facilitating the processing of the selected item.

How might these selection mechanisms operate? As alluded to in the introduction, attentional selection could operate in several different ways. One possibility is that attentional selection mechanisms suppress inputs from neighboring items. As outlined by Luck et al. (1997), such an active inhibition of neighboring items would alleviate potential ambiguities in perceptual coding. However, such active inhibitory mechanisms are not the only way such localized performance decrements might arise. Bahcall and Kowler (1999) proposed that processing at an attended location could be augmented by "borrowing" processing resources from neighboring locations. This in essence decreases the processing capacity allotted to neighboring locations, although no active inhibitory mechanisms are involved. Bahcall and Kowler's model can be considered a space-based model of attention; however, it is not difficult to envision its object-based counterpart. If we assume that attentional processing resources are spatially dependent, then an attentional salience-based competition for control of these processing resources would also yield the observed pattern of inhibition. Items neighboring an attentionally salient item would share a greater proportion of processing resources with the salient item than would more distant items. As a result of the competition for these processing resources, items neighboring an attentionally salient item recruit fewer processing resources than items more distant from the salient item.

\section{Conclusions}

The present study provides further evidence that one consequence of attending to an object is the inhibited processing of neighboring objects. The present study extends this finding to the reaction time domain and demon- 
strates that this inhibition is susceptible to top-down control in the form of attentional set. Finally, these results (and others like them) are placed in the broader context of how various attentional mechanisms modulate perceptual processing. This analysis suggests that researchers need to be cognizant of the different objectives of attention (e.g., preparation vs. selection) and possible differences in the underlying mechanisms.

\section{REFERENCES}

Bacon, W. F., \& Egeth, H. E. (1994). Overriding stimulus-driven attentional capture. Perception \& Psychophysics, 55, 485-496.

Bahcall, D. O., \& Kowler, E. (1999). Attentional interference at small spatial separations. Vision Research, 39, 71-86.

Caputo, G., \& Guerra, S. (1998). Attentional selection by distractor suppression. Vision Research, 38, 669-689.

CAve, K. R., \& Zimmerman, J. M. (1997). Flexibility in spatial attention before and after practice. Psychological Science, 8, 399-403.

Chastain, G. (1992). Is rapid performance improvement across short precue-target delays due to masking from peripheral precues? Acta Psychologica, 79, 101-114.

Cheal, M. L. (1993). Rapid performance improvement across short precue-target delays is most likely due to effects of attention and not to masking from peripheral precues: A comment on Chastain, 1992. Acta Psychologica, 83, 79-86.

CheAL, M. L. (1997). Understanding diverse effects of visual attention with the VAP-filters metaphor. Consciousness \& Cognition, 6, 348-362.

Cheal, M. L., \& Lyon, D. R. (1992). Benefits from attention depend on the target type in location-precued discrimination. Acta Psychologica, 81, 243-267.

Cheal, M. L., Lyon, D. R., \& GotTlob, L. R. (1994). A framework for understanding the allocation of attention in location-precued discrimination. Quarterly Journal of Experimental Psychology, 47A, 699-739.

Chelazzi, L., Miller, E. K., DunCan, J., \& Desimone, R. (1993). A neural basis for visual search in inferior temporal cortex. Nature, 363, 345-347.

Desimone, R., \& DunCan, J. (1995). Neural mechanisms of selective attention. Annual Review of Neuroscience, 18, 193-222.

Desimone, R., Wessinger, M., Thomas, L., \& Schneider, W. (1990). Attentional control of visual perception: Cortical and subcortical mechanisms. Cold Spring Harbor Symposium on Quantitative Biology, 55, 963-971.

Downing, C. J. (1988). Expectancy and visual-spatial attention: Effects on perceptual quality. Journal of Experimental Psychology: Human Perception \& Performance, 14, 188-202.

Downing, C. J., \& Pinker, S. (1985). The spatial structure of visual attention. In M. I. Posner \& O. S. M. Marin (Eds.), Attention and performance XI (pp. 171-188). Hillsdale, NJ: Erlbaum.

DunCAN, J., \& Humphreys, G. (1989). Visual search and stimulus similarity. Psychological Review, 96, 433-458.

EGETH, H. E., VirZI, R. A., \& GARBART, H. (1984). Searching for conjunctively defined targets. Journal of Experimental Psychology: Human Perception \& Performance, 10, 32-39.

ERIKSEN, C. W., \& Hoffman, J. E. (1972). Temporal and spatial characteristics of selective encoding from visual displays. Perception \& Psychophysics, 12, 201-204.

Fol. K, C. L., \& Remington, R. W. (1998). Selectivity in distraction by irrelevant featural singletons: Evidence for two forms of attentional capture. Journal of Experimental Psychology: Human Perception \& Performance, 24, 847-858.

Folk, C. L., Remington, R. W., \& Johnston, J. C. (1992). Involuntary covert orienting is contingent on attentional control settings. Journal of Experimental Psychology: Human Perception \& Performance, 18, 1030-1044.

Hawkins, H. L., Hillyard, S. A., Luck, S. J., Mouloua, M., Down-
ING, C. J., \& Woodwarn, D. P. (1990). Visual attention modulates signal detectability. Journal of Experimental Psychology: Human Perception \& Performance, 16, 802-811.

Henderson, J. M., \& Macquistan, A. D. (1993). The spatial distribution of attention following an exogenous cue. Perception \& Psychophysics, 53, 221-230.

JONIDES, J., \& YANTIS, S. (1988). Uniqueness of abrupt visual onset in capturing attention. Perception \& Psychophysics, 43, 346-354.

Kaptein, N. A., Theeuwes, J., \& van der Heijden, A. H. C. (1995). Search for a conjunctively defined target can be selectively limited to a color-defined subset of elements. Journal of Experimental Psychology: Human Perception \& Performance, 21, 1053-1069.

LABERGE, D. (1995). Attentional processing: The brain's art of mindfulness. Cambridge, MA: Harvard University Press.

LABERGE, D., \& Brown, V. (1989). Theory of attentional operations in shape identification. Psychological Review, 96, 101-124.

LaBerge, D., Carlson, R. L., Williams, J. K., \& Bunney, B. G. (1997). Shifting attention in visual space: Tests of moving-spotlight models versus an activity-distribution model. Journal of Experimental Psychology: Human Perception \& Performance, 23, 1380-1392.

Luck, S. J., Girelli, M., McDermotT, M. T., \& Ford, M. A. (1997). Bridging the gap between monkey neurophysiology and human perception: An ambiguity resolution theory of visual selective attention. Cognitive Psychology, 33, 64-87.

MoRAN, J., \& DESIMONE, R. (1985). Selective attention gates visual processing in the extrastriate cortex. Science, 229, 782-784.

MotTER, B. C. (1993). Focal attention produces spatially selective processing in visual cortical areas V1, V2, and V4 in the presence of competing stimuli. Journal of Neurophysiology, 70, 909-919.

MounTS, J. R. W. (2000). Evidence for suppressive mechanisms in attentional selection: Feature singletons produce inhibitory surrounds. Perception \& Psychophysics, 62, 969-983.

Nakayama, K., \& Mackeben, M. (1989). Sustained and transient components of focal visual attention. Vision Research, 29, 1631-1647.

PoSNer, M. I., SNyder, C. R., \& Davidson, B. J. (1980). Attention and the detection of signals. Journal of Experimental Psychology: General, 109, 160-174.

THEEUWES, J. (1991). Exogenous and endogenous control of attention: The effect of visual onsets and offsets. Perception \& Psychophysics, 49, 83-90.

TheEUWEs, J. (1992). Perceptual selectivity for color and form. Perception \& Psychophysics, 51, 599-606.

YANTIS, S. (1996). Attentional capture in vision. In A. F. Kramer, M. G. H. Coles, \& G. D. Logan (Eds.), Converging operations in the study of visual selective attention (pp. 45-76). Washington, DC: American Psychological Association.

YANTIS, S., \& EGETH, H. E. (1999). On the distinction between visual salience and stimulus-driven attentional capture. Journal of Experimental Psychology: Human Perception \& Performance, 25, 661-676.

Yantis, S., \& Hillstrom, A. P. (1994). Stimulus-driven attentional capture: Evidence from equiluminant visual objects. Journal of Experimental Psychology: Human Perception \& Performance, 20, 95-107.

YANTIS, S., \& JoNiDES, J. (1984). Abrupt visual onsets and selective attention: Evidence from visual search. Journal of Experimental Psychology: Human Perception \& Performance, 10, 601-621.

\section{NOTE}

1. Caputo and Guerra (1998) measured RTs as well as line length thresholds in their Experiment 3 and found evidence for inhibition in the RT data as well. However, these RTs were for near-threshold discriminations. The present study sought to extend these findings to suprathreshold stimulus differences.

(Manuscript received July 20, 1999; revision accepted for publication December 9, 1999.) 